\title{
Short-term mechanical circulatory support as bridge to heart transplantation: paracorporeal ventricular assist device as alternative to extracorporeal life support
}

\author{
Sandro Sponga, Giovanni Benedetti, Ugolino Livi \\ Cardiothoracic Department, University Hospital of Udine, Udine, Italy \\ Correspondence to: Giovanni Benedetti. Cardiothoracic Department, University Hospital of Udine, P.le S.M. della Misericordia, 15, Udine 33100, \\ Italy. Email: giovanni.benedetti@asuiud.sanita.fvg.it.
}

\begin{abstract}
Extracorporeal life support (ECLS) is generally considered to be the treatment of choice for bridging to heart transplantation (HTx) patients with cardiogenic shock; however, alternative mechanical circulatory support (MCS) devices have been proposed with satisfactory results and, among those, paracorporeal systems have demonstrated to be safe and effective. This technology has been used for decades as bridge to transplant, especially in patients with advanced right ventricular dysfunction or evidence of multiorgan failure (MOF), which could be difficult to manage with an isolated left ventricular support. Paracorporeal systems are defined by having the pump located outside of the body, with inflow and outflow cannulas that traverse the skin connecting the pump with the heart and great vessels. They can be utilised in a uni- or biventricular configuration and can provide pulsatile or continuous flow, depending on the device technology (pneumatic vs. centrifugal). In particular, pneumatic devices allow for patient mobilization and hospital discharge, improving rehabilitation and organ recovery while bridging to transplant. In our institution at the University Hospital of Udine, 34 pneumatic paracorporeal ventricular assist devices (VADs) have been implanted since 1998: in most cases (32 pts), as biventricular support for patients in INTERMACS class I-II. After a median support time of 34 (range, 0-385) days, with 19 patients (56\%) supported for more than 1 month, 23 patients (68\%) underwent HTx and $3(9 \%)$ were successfully weaned to hospital discharge, resulting in an overall combined $76 \%$ survival to $\mathrm{HTx}$ or weaning. After transplant, the survival rate was similar to the one of the patients not bridged with MCS. In conclusion, pneumatic VADs can effectively assist patients with severe biventricular failure, especially those with contraindications to ECLS or expected long waiting times for HTx. Moreover, they can potentially result in hospital discharge, optimal organ and patient recovery and donor-recipient matching, resulting in a satisfactory transplant outcome.
\end{abstract}

Keywords: Bridge to transplant; BVAD; cardiogenic shock

Submitted Dec 29, 2018. Accepted for publication Dec 31, 2018.

doi: 10.21037 /acs.2019.01.01

View this article at: http://dx.doi.org/10.21037/acs.2019.01.01

\section{Introduction}

Patients with advanced heart failure refractory to standard medical treatment have a poor prognosis and quality of life. Despite intensive care and inotropic support, progression to cardiogenic shock and consequent multiorgan failure increases patient mortality while awaiting heart transplantation (HTx). Furthermore, when HTx is performed in this urgent/emergent setting, the outcome is generally worse than usual (1). Extracorporeal life support (ECLS), a short-term mechanical circulatory support (MCS) modality, has been suggested as a treatment of choice in cardiogenic shock because it provides, at the same time, both pulmonary and circulatory support, and because of its limited surgical invasiveness, easy implantation and ready availability $(2,3)$. Despite this, ECLS support is burdened by a significant risk of 30-day mortality and morbidity 
such as bleeding, stroke, infection and ineffective left ventricular unloading (2-5). A variety of temporary MCS devices and implantation strategies have been proposed as alternatives to ECLS, and each of those presents advantages and disadvantages. Intracorporeal percutaneous devices (Impella $^{\circledR}$ and Tandem Heart ${ }^{\circledR}$ ) and paracorporeal devices (centrifugal and pneumatic pumps) are the most commonly utilized solutions.

\section{History of paracorporeal MCS devices}

DeBakey in the 1960s was the first to describe the application of a paracorporeal, pneumatically driven pump as a post-cardiotomy ventricular assist device (VAD) to wean the patient from the cardiopulmonary bypass and wait for the heart to recover. In the following decades, as HTx became a reality, the perspective of MCS also changed by being used as bridge to transplantation. At the time, available technology consisted of large consoles and extra- or paracorporeal pumps, not compatible with hospital discharge. The first successful case surviving the paracorporeal VAD implantation was reported by Hill et al. in 1986 (6). Since then, MCS underwent a rapid, worldwide spread, mainly as a bridge to HTx. Since VADs became widely used, research and technology have enabled for the progressive miniaturization of pumps and control units, while reducing the rate of adverse events, obtaining a longer and safer support. The long-term outcome of patients supported by VAD has improved continuously in recent years and accordingly, VADs have also been used as destination therapy, representing an alternative to transplantation. Smaller, safer devices are changing the VAD panorama, narrowing the indications for paracorporeal systems in adult patients (7).

\section{Paracorporeal devices}

Paracorporeal systems have the pump located outside the body, with inflow and outflow cannulas that traverse the skin connecting the pump with the heart and great vessels. They can be utilized to support the left, right or both ventricles. Among paracorporeal devices, centrifugal pumps are composed of a rotating impeller that propels the blood located inside the pump, whilst the energy source is outside the blood chamber (rotor). Pump flow is directly proportional to pump rotor speed and inversely proportional to pressure difference between the inlet and outlet orifice, thus is pre- and after-load sensitive. It can be used to support the patient's circulation for days or weeks, as a bridge to transplantation, to recovery or to further VAD implantation. Possible complications related to centrifugal pump technology include bleeding, hemolysis or mechanical obstruction by tube kinking during patient mobilization. Unfortunately, no published studies are currently available that compare these centrally-cannulated pumps with other temporary MCS options, but most clinicians would lean towards the use of these devices in the postcardiotomy setting.

Pulsatile pumps are usually composed of a rigid case containing a membrane that separates blood and air. The blood is propelled by positive displacement, by cyclically changing the internal volume of the compression chamber through a pneumatically-driven expansion of the membrane, directing the flow with one-way valves. The flow can be determined by setting rate and percentage of the chamber systole and by regulating preload and afterload. The energy source is an external compressor, which in more recent models has become smaller and portable. Two devices are available for pneumatic paracorporeal support: Thoratec PVAD and Excor Berlin Heart.

\section{Thoratec PVAD (Thoratec Corp., Pleasanton, CA, USA)}

This pneumatic pump, which provides long-term unior biventricular support, is positioned on the patient's abdomen with the cannulae crossing the skin to reach the mediastinum. It consists in a flexible blood bladder of $65 \mathrm{~mL}$ volume made of Thoralon (a material that seems to minimize blood clotting and inflammation), controlled by a dual-drive console which alternates negative and positive air pressure. Mechanical valves (Monostrut tilting Delrin disc) on the inflow and outflow maintain unidirectional blood flow. The stroke volume is fixed, so the pump rate must be adjusted to modulate the pump output. It is designed to provide longterm biventricular support, but the dimensions of the console severely impact on the mobility of the patient, who can ambulate but usually can't leave the hospital. Usually, its use is precluded in children due to its size.

\section{Berlin Heart EXCOR (BerlinHeart, Berlin, Germany)}

This paracorporeal VAD is available in various pump volume sizes $(10,25,30,50,60,80 \mathrm{~mL})$, suitable to support patients from a neonatal age through to adulthood. It can be implanted as either uni- or biventricular support, consisting 


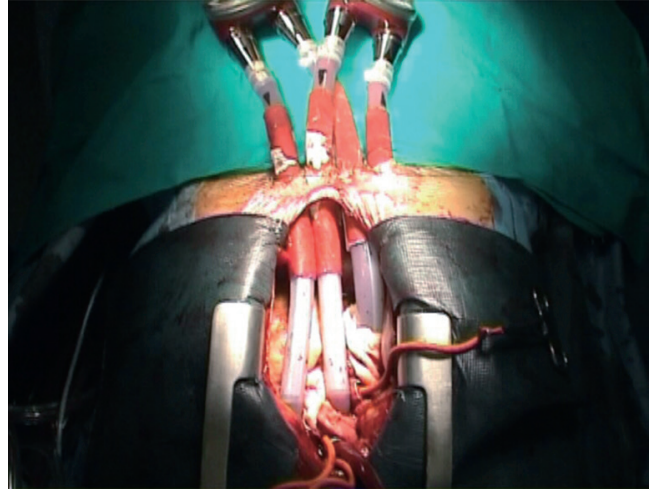

Figure 1 BVAD implantation.

of a pneumatic device made of a three-layered flexible polyurethane membrane encased within a transparent semirigid housing, for better detection of thrombus formation. Between the membranes lies a graphite powder lubricant to minimize their friction and additionally, the innermost membrane, which is in contact with blood, is heparincoated (8). The adult sizes were provided with mechanical mono-leaflet tilting disc valves, but now they have heparincoated polyurethane valves as is in the case of pediatric valves. Also, the cannulas are available in small diameters and the drive units are wearable thanks to rechargeable batteries, allowing for patient mobility.

\section{Indications}

Paracorporeal VADs had a leading role as medium- to long-term assist devices in refractory heart failure, but their importance has decreased since the late 1990s due to the progressive diffusion of LVADs and extracorporeal membrane oxygenation (ECMO) (9). However, these pumps have still an indisputable indication in neonatal or pediatric patients, whose body size precludes the fitting of an implantable pump. Patients in INTERMACS level 1 or 2 with biventricular heart failure represent high risk MCS candidates. According to the last INTERMACS report, one-year survival for these patients is in the range of 50-60\% (BVAD paracorporeal or LVAD plus centrifugal pump) (7). Although it is desirable to implant an LVAD, there are a considerable number of patients who require primary BVAD support. This accounts for $10-20 \%$ of the entire VAD population in some reports (10). In addition, Takeda et al. found that patients receiving unplanned secondary RV support had much lower survival chances (11).
As depicted in the INTERMACS report (7), paracorporeal pumps play a definite role in patients with contraindications to ECLS because of peripheral vasculopathy, bleeding or contraindications to heparin infusion.

Another category of patients for which paracorporeal VADs might be beneficial is those affected by a reversible cause of cardiogenic shock, such as viral or drug-related myocarditis. In these patients, biventricular failure is common and paracorporeal devices, if confronted with ECLS, can guarantee a longer support increasing the chances of myocardial recovery. Other patients that can benefit by this treatment are those who would have to wait longer on the transplant list on circulatory support because of large body surface areas, more rare blood groups, severe immunosensitization, or systemic and tumoral disease with a not yet defined prognosis. Indications for BVAD support can be summarized as follows:

* Acute cardiogenic shock with multi-organ dysfunction;

* Intractable ventricular arrhythmia or persistent ventricular fibrillation;

* Severe right heart failure on inotropic support;

* Acute myocarditis;

* Acute biventricular myocardial infarction.

\section{Surgical technique and management}

Surgical setup includes transesophageal echocardiography for cardiac visualization and implantation guidance, general anesthesia and full median sternotomy. Extracorporeal circulation is established through bicaval venous and aortic cannulation (slightly more distally in the aortic arch), and the VAD implantation is done off-clamp. In case of redo surgery, right subclavian cannulation should be preferred. The BiVAD implantation follows typically these steps: LV apex (or left atrium), right atrium, main pulmonary artery, ascending aorta (Figure 1). Left atrial cannulation is preferred in patients affected by hypertrophic or restrictive cardiomyopathy with very small LV cavity, or presenting a large apical myocardial infarction. The anastomosis of the outflow cannulas to the PA and the ascending aorta are performed after side bite clamping.

Early postoperative management includes monitoring blood pressure, pulmonary pressure, peripheral oxygen saturation and fluid balance measuring. Furthermore, VAD output, rate, vacuum and fill setting to reach complete emptying of the chambers all must be monitored. Management of bleeding includes meticulous haemostasis 
and sternal closure, aggressive correction of hematologic abnormalities and acidosis, a warming blanket to prevent hypothermia and early re-exploration if needed. Infection in these patients is a constant concern, especially at the cannulas exit site: if needed, it can be treated with local antibiotic irrigation and, in more complex cases, IV antibiotics could be applied. Nutritional and psychological supports are crucial, including education of both patient and caregiver alongside active family involvement.

\section{Outcomes}

While many different devices have been used in the past decades, only 2 devices are now routinely used around the world for pneumatic paracorporeal support: Thoratec PVAD and Berlin Heart EXCOR. There isn't any scientific evidence that support the use of one of the two devices over the other, so the choice is largely based on local experience.

The INTERMACS Registry reports that survival with BiVAD support has remained inferior to that of isolated LVAD (7) and, in the most recent era, only about $50 \%$ of patients were alive at 1 year, just slightly better than early and mid-term results of a total artificial heart. As of 2016, Thoratec PVAD has been implanted in around 5,000 patients $(56 \%$ BiVAD, 36\% LVAD, 6\% RVAD patients) in 277 centers worldwide. The longest duration of support was 1,276 days (3.4 years) and different studies have shown a survival to transplantation of $70 \%(7,12,13)$. A single-center experience at Cedars Sinai (USA) reported 80 INTERMACS 1 patients (cardiogenic shock) treated with a BVAD as a bridge to transplantation between 2000 and 2008 (14). Survival to transplantation was $71.3 \%$ with a significantly better outcome in the late years (survival to transplantation after 2006 was $82 \%$ ). The rate of clinically significant thromboembolic complications was relatively low (15\%), while no clinically significant infection or VAD endocarditis was reported.

Since 1985 to date, Berlin Heart EXCOR has been successfully used in more than 2,200 patients at 106 heart centers in 29 countries worldwide $(7,12,13)$. The main indications were dilated cardiomyopathy, ischemic cardiomyopathy and acute myocardial infarction. The preferred configuration was BVAD (68\%), followed by LVAD (29\%) and RVAD (3\%). The mean time on device was 137 days and longest time on device $>5$ years, with an overall 1-year survival of $76 \%$, with $47 \%$ of patients being transplanted. With more experience, better outcome for patients on EXCOR BVAD support have been accomplished during recent years, with a survival rate of more than $90 \%$ in selected younger patients supported by EXCOR BVAD (15).

Some institutions have reported a satisfactory 30-day mortality following BVAD implantation in selected patients: Potapov et al. showed a 33\%-mortality in their singlecenter experience on patients with very advanced heart failure (16), and Tsukui et al. an even lower mortality of $15 \%$ in patients bridged to HTx (17). A recent study from the University of Heidelberg on patients in INTERMACS 1-2 reports a 1 -year survival of $92 \%$ (18), while Creaser et al. (19) suggest that BVAD patients can be discharged safely under close management of multidisciplinary team while awaiting for a transplant. Other authors, on the other hand, have suggested that use of BVAD portends an increased risk of both early and late post-transplant mortality (20-23).

\section{Udine experience}

In our unit at the University Hospital of Udine, 129 patients affected by advanced heart failure of different aetiologies were mechanically supported with short term MCS since 1998. Among them, 34 were Berlin Heart Excor paracorporeal devices (Berlin Heart GmbH, Berlin, Germany) and in most cases (32 pts) a biventricular support was adopted. Intracorporeal LVAD implantation was excluded because of severe hemodynamic impairment involving both ventricles. All these patients were in critical conditions, $62 \%$ of them were in INTERMACS class $1,53 \%$ needed IABP support and $21 \%$ mechanical ventilation (MV), 35\% were affected by renal failure and $24 \%$ by liver dysfunction (Table 1). The median age of the patients was $54.9(14.8-68.3), 97 \%$ were male and the most frequent cause of heart failure were ischemic and dilated cardiomyopathy (41\%).

After implantation, 3 patients (9\%) died because of early multi organ failure (MOF). An additional 5 patients died after 1 month while in hospital: 2 for progression of MOF, 1 for cerebral ischemia, 1 for sepsis and 1 because of late cardiac tamponade. Fifty-nine percent of patients needed sternal re-exploration for post-operative bleeding, 32\% experienced device-related infection and $9 \%$ had pump thrombosis. Other common complications were acute kidney injury (AKI) in $32 \%$ of patients, needing continuous renal replacement therapy (CRRT) in 29\% and liver dysfunction in $24 \%$. The median hospital stay was 45 (range, 0-146) days. After a median support time of 34 (range, 
Table 1 Preoperative patient characteristics

\begin{tabular}{|ll}
\hline Demographics & $\mathrm{n}[\%], \mathrm{N}=34$ \\
\hline Age (years, median, range) & $54.9(14.8-68.3)$ \\
\hline Female sex & $1[3]$ \\
\hline BMI (kg/m², mean $\pm \mathrm{SD})$ & $25 \pm 4.6$ \\
\hline BSA (mean \pm SD) & $1.9 \pm 0.2$ \\
\hline Etiology & \\
\hline Ischemic cardiopathy & $14[41]$ \\
\hline Dilated cardiomyopathy & $14[41]$ \\
\hline Myocarditis & $4[12]$ \\
\hline Other & $2[6]$ \\
\hline Diabetes & $9[26]$ \\
\hline Hyperlipidemia & $11[32]$ \\
\hline Hypertension & $9[26]$ \\
\hline COPD & $3[9]$ \\
\hline Renal failure & $12[35]$ \\
\hline Liver dysfunction & $8[24]$ \\
\hline Peripheral vascular disease & $2[6]$ \\
\hline Atrial fibrillation & 8 obstructive pulmonary disease. \\
\hline BMI, body mass index; & \\
\hline & \\
\hline
\end{tabular}

0-385) days, with 19 patients (56\%) having a support longer than 1 month, 23 patients (68\%) were bridged to HTx and $3(9 \%)$ were successfully weaned with hospital discharge, resulting in an overall combined survival rate of $77 \%$ (Table 2). Considering the overall survival of patients requiring paracorporeal $\mathrm{VAD}$ and then transplanted or weaned, the 1-, 5- and 10-year survival was $67 \pm 8,60 \pm 9$ and $50 \pm 10$ months during an overall median follow-up of 21 (range, 0-240) months.

\section{Discussion}

During the last decade, intracorporal implantable LVADs have been widely adopted as a bridge to HTx. Unfortunately, these devices are not the best therapeutic option when the patient is in cardiogenic shock showing biventricular involvement and initial multiorgan dysfunction (24-26).
Table 2 Complications and outcomes after pneumatic paracorporeal BVAD implantation

\begin{tabular}{|c|c|}
\hline Complications & $\mathrm{n}[\%], \mathrm{N}=34$ \\
\hline Device-related infection & $11[32]$ \\
\hline AKI & $11[32]$ \\
\hline CRRT & 10 [29] \\
\hline Liver dysfunction & $8[24]$ \\
\hline Atrial fibrillation & $5[15]$ \\
\hline Ischemic stroke & $1[3]$ \\
\hline Pump thrombosis & 3 [9] \\
\hline Mediastinitis & $2[6]$ \\
\hline Sternal re-exploration & $20[59]$ \\
\hline \multicolumn{2}{|l|}{ Outcomes } \\
\hline $\begin{array}{l}\text { Median time of device assistance, } \\
\text { days (range) }\end{array}$ & $34(0-385)$ \\
\hline Early mortality & 3 [9] \\
\hline In-hospital mortality & 8 [24] \\
\hline Median hospital stay, days (range) & $45(0-146)$ \\
\hline Patients transplanted & 23 [68] \\
\hline Weaning & $3[9]$ \\
\hline \multicolumn{2}{|c|}{$\begin{array}{l}\text { AKI, acute kidney injury; CRRT, continuous renal replacement } \\
\text { therapy. }\end{array}$} \\
\hline
\end{tabular}

ECLS has the advantage of being readily implanted in most patients and different clinical scenarios but is burdened with a significant mortality and morbidity rate. Moreover, to obtain effective left ventricle unloading, high-dose inotropic support is often necessary, alongside IABP and/or $\mathrm{LV}$ venting, with the result of increasing the risk of local or systemic complications $(2,3,5,27)$.

Among paracorporeal devices, centrifugal pumps are commonly used because they are relatively cheap and simple to manage compared to other devices. The absence of the oxygenator can potentially lower the impact of inflammation and coagulation imbalance and offer a more physiologic blood flow both in uni- or biventricular configurations. Despite a more demanding surgical approach, pneumatic paracorporeal devices may represent an appealing alternative to ECLS, not only by reducing the oxygenator-related complications, but also by 
offering a longer circulatory support compared to ECLS and centrifugal pumps, allowing thereby a safer organ donor allocation and a better multiorgan recovery and preservation: indeed, long-term circulatory support can improve end-organ function, even to complete recovery and permit the optimization of a patient's fitness and nutritional status before HTx.

It has also been demonstrated that pulsatile flow plays a significant role in preserving the microcirculation of patients affected by cardiogenic shock by improving endorgan tissue perfusion and capillary flow as measured in the liver, kidneys and stomach $(28,29)$. Also, continuous flow was associated with an increased likelihood of damaging the Von Willebrand factor (30). Cardiogenic shock causes gastrointestinal mucosal ischemia, which increases the risk of bacterial translocation and of ischemic-hemorrhagic major events. In this setting, pulsatile flow could be advantageous over continuous flow, both by improving the mucosal capillary perfusion and avoiding the acquired Von Willebrand Syndrome.

On the other hand, many centers avoid using BVAD support as a bridge to HTx because of a more demanding implantation technique, increased HTx risk due to a previous sternotomy and major costs including overall hospital resources utilization (31). Moreover, as confirmed in our own experience, perioperative bleeding or late cardiac tamponade might be a serious complication and require a careful patient management and monitoring. Device failure and pump thrombosis are potentially catastrophic issues, although fortunately are not very frequent: the reported incidence is around 5\% (32) and it occurred in 3 patients of our cohort, requiring in one case a pump exchange 2 months after implantation.

Three of our patients have been discharged home for 2, 3 and 11 months respectively and then successfully transplanted, accounting for the possibility of a long period of assistance to get an ideal patient recovery and donor organ matching (33). Considering our results in terms of patient survival up to HTx or weaning, it is remarkable the percentage of terminally ill patients who has benefited from pneumatic paracorporeal support, is not much dissimilar to that obtained with LVAD in cases with less advanced heart failure.

\section{Conclusions}

Patients presenting with refractory advanced heart failure are a demanding population which require intensive treatment and more often circulatory MCS to prevent irreversible multiorgan dysfunction. Despite the advent of new, smaller and safer devices, paracorporeal VADs still have a role in pediatric cases and in selected adult patients. In case of biventricular failure, these devices have to be considered for a short- to medium-term bridging, with satisfactory outcomes at follow-up. In our experience, pneumatic paracorporeal VAD can be considered a valid alternative of ECLS, providing an effective support as a bridge to HTx. Although surgical BVAD implantation may be more demanding and associated with the risk of perioperative bleeding, such therapeutic choice can potentially allow for complete organ recovery and improve patient rehabilitation, to achieve the best outcome of HTx.

\section{Acknowledgements}

We thank Veronica Ferrara e Helena Hortis for data collection and analysis.

\section{Footnote}

Conflicts of Interest: The authors have no conflicts of interest to declare.

\section{References}

1. Mohite PN, Zych B, Banner NR, et al. Refractory heart failure dependent on short-term mechanical circulatory support: what next? Heart transplant or long-term ventricular assist device. Artif Organs 2014;38:276-81.

2. Rastan AJ, Dege A, Mohr M, et al. Early and late outcomes of 517 consecutive adult patients treated with extracorporeal membrane oxygenation for refractory postcardiotomy cardiogenic shock. J Thorac Cardiovasc Surg 2010;139:302-11, 311.e1.

3. Combes A, Leprince P, Luyt CE, et al. Outcomes and long-term quality-of-life of patients supported by extracorporeal membrane oxygenation for refractory cardiogenic shock. Crit Care Med 2008;36:1404-11.

4. Werdan K, Gielen S, Ebelt H, et al. Mechanical circulatory support in cardiogenic shock. Eur Heart J 2014;35:156-67.

5. Hoefer D, Ruttmann E, Poelzl G, et al. Outcome evaluation of the bridge-to-bridge concept in patients with cardiogenic shock. Ann Thorac Surg 2006;82:28-33.

6. Hill JD, Farrar DJ, Hershon JJ, et al. Use of a prosthetic ventricle as a bridge to cardiac transplantation for postinfarction cardiogenic shock. N Engl J Med 
1986;314:626-8.

7. Kirklin JK, Pagani FD, Kormos RL, et al. Eighth annual INTERMACS report: Special focus on framing the impact of adverse events. J Heart Lung Transplant 2017;36:1080-6.

8. Werkkala K, Jokinen JJ, Soininen L, et al. Clinical durability of the CARMEDA® BioActive Surface in EXCOR® VAD pumps. ASAIO J 2016;62:139-42.

9. Lahpor J, Khaghani A, Hetzer R, et al. European results with a continuous-flow ventricular assist device for advanced heart-failure patients. Eur J Cardiothorac Surg 2010;37:357-61.

10. Boulate D, Marques MA, Ha R, et al. Biventricular VAD versus $\mathrm{LVAD}$ for right heart failure. Ann Cardiothorac Surg 2014;3:585-8.

11. Takeda K, Naka Y, Yang JA, et al. Outcome of unplanned right ventricular assist device support for severe right heart failure after implantable left ventricular assist device insertion. J Heart Lung Transplant 2014;33:141-8.

12. Kirklin JK, Xie R, Cowger J, et al. Second annual report from the ISHLT Mechanically Assisted Circulatory Support Registry. J Heart Lung Transplant 2018;37:685-91.

13. de By TMMH, Mohacsi P, Gahl B, et al. The European Registry for Patients with Mechanical Circulatory Support (EUROMACS) of the European Association for Cardio-Thoracic Surgery (EACTS): second report. Eur J Cardiothorac Surg 2017. [Epub ahead of print].

14. Moriguchi J, Davis S, Jocson R, et al. Successful use of a pneumatic biventricular assist device as a bridge to transplantation in cardiogenic shock. J Heart Lung Transplant 2011;30:1143-7.

15. Bartfay SE, Liden H, Holmberg M, et al, The BiVAD experience at the university hospital, results from the years 2010 to 2012 and comparison with contemporary LVAD patients. Eur Heart J 2013;34:P2182.

16. Potapov EV, Stepanenko A, Kukucka M, et al. Prediction of survival in patients with cardiogenic shock and multiorgan failure treated with biventricular assist device. ASAIO J 2010;56:273-8.

17. Tsukui H, Teuteberg JJ, Murali S, et al. Biventricular assist device utilization for patients with morbid congestive heart failure: a justifiable strategy. Circulation 2005;112:I65-72.

18. Schmack B, Weymann A, Ruschitzka F, et al. Successful support of biventricular heart failure patients by new EXCOR $®$ Adult pumps with bileaflet valves: a prospective study. Clin Res Cardiol 2018;107:413-20.

19. Creaser JW, Rourke D, Vandenbogaart E, et al. Outcomes of biventricular mechanical support patients discharged to home to await heart transplantation. J Cardiovasc Nurs 2015;30:E13-20.

20. Patlolla V, Patten RD, Denofrio D, et al. The effect of ventricular assist devices on post-transplant mortality an analysis of the United network for organ sharing thoracic registry. J Am Coll Cardiol 2009;53:264-71.

21. Alba AC, McDonald M, Rao V, et al. The effect of ventricular assist devices on long-term post-transplant outcomes: a systematic review of observational studies. Eur J Heart Fail 2011;13:785-95.

22. Barth E, Durand M, Heylbroeck C, et al. Extracorporeal life support as a bridge to high-urgency heart transplantation. Clin Transplant 2012;26:484-8.

23. Reser D, Fröhlich GM, Seifert B, et al. The impact of pretransplantation urgency status and the presence of a ventricular assist device on outcome after heart transplantation. Transplant Proc 2014;46:1463-8.

24. Aissaoui N, Morshuis M, Paluszkiewicz L, et al. Comparison of biventricular and left ventricular assist devices for the management of severe right ventricular dysfunction in patients with end-stage heart failure. ASAIO J 2014;60:400-6.

25. Cleveland JC Jr, Naftel DC, Reece TB, et al. Survival after biventricular assist device implantation: an analysis of the Interagency Registry for Mechanically Assisted Circulatory Support database. J Heart Lung Transplant 2011;30:862-9.

26. Nagpal AD, Singal RK, Arora RC, et al. Temporary Mechanical Circulatory Support in Cardiac Critical Care: A State of the Art Review and Algorithm for Device Selection. Can J Cardiol 2017;33:110-8.

27. Bakhtiary F, Keller H, Dogan S, et al. Venoarterial extracorporeal membrane oxygenation for treatment of cardiogenic shock: clinical experiences in 45 adult patients. J Thorac Cardiovasc Surg 2008;135:382-8.

28. Orime Y, Shiono M, Nakata K, et al. The role of pulsatility in end-organ microcirculation after cardiogenic shock. ASAIO J 1996;42:M724-9.

29. Sezai A, Shiono M, Orime Y, et al. Major organ function under mechanical support: comparative studies of pulsatile and nonpulsatile circulation. Artif Organs 1999;23:280-5.

30. Sponga S, Nalli C, Casonato A, et al. Severe upper gastrointestinal bleeding in Heartmate II induced by acquired von Willebrand deficiency: anticoagulation management. Ann Thorac Surg 2012;94:e41-3.

31. Bartfay SE, Dellgren G, Lidén H, et al. Are biventricular assist devices underused as a bridge to heart transplantation in patients with a high risk of postimplant right ventricular failure? J Thorac Cardiovasc Surg 2017;153:360-7.e1. 
32. Barge-Caballero E, Almenar-Bonet L, Villa-Arranz A, et al. Impact of short-term mechanical circulatory support with extracorporeal devices on postoperative outcomes after emergency heart transplantation: data from a multi-

Cite this article as: Sponga S, Benedetti G, Livi U. Shortterm mechanical circulatory support as bridge to heart transplantation: paracorporeal ventricular assist device as alternative to extracorporeal life support. Ann Cardiothorac Surg 2019;8(1):143-150. doi: 10.21037/acs.2019.01.01 institutional Spanish cohort. Int J Cardiol 2014;176:86-93.

33. Sponga S, Bagur R, Livi U. Teleconsultation for left ventricular assist device patients: a new standard of care. Eur J Heart Fail 2018;20:818-21. 